\title{
THE SEPARATION PRINCIPLE FOR IMPULSE CONTROL PROBLEMS
}

\author{
JOSE-LUIS MENALDI
}

\begin{abstract}
In this paper, one shows that the combined problem of optimal impulse control and filtering, for a stochastic linear dynamic system observed via a noisy linear channel, can be reduced to two independent problems of impulse control and filtering, respectively.
\end{abstract}

1. Introduction. W. M. Wonham [8] showed that the combined problem of optimal control and filtering, for a stochastic linear dynamic system observed via a noisy linear channel, can be reduced to two independent problems of stochastic control and filtering, respectively. This result was improved by M. H. A. Davis [3] using the concept of Girsanov solutions of stochastic differential equations.

A. Bensoussan and J. L. Lions [1] proved that the same separation principle holds for stopping time problems.

In all cases, a nondegeneracy on the observation matrix is imposed. This assumption would rarely be met in practice.

In [5], we showed that the separation principle for stopping time problems holds even under degeneracy.

Let us also mention the work of J. Szpirglas and G. Mazziotto [7].

The object of this article is to prove that the combined problem of optimal impulse control and filtering, for a stochastic linear dynamic system observed via a noisy linear channel, can be reduced to two independent problems of impulse control and filtering, respectively. In general, the optimal impulse control depends parametrically on the intensity of channel noise; the result means, however, that channel noise plays qualitatively the same role as dynamic disturbances in determination of the feedback law.

2. Statement of the problem. Let $(\Omega, \mathscr{F}, P)$ be a probability space and $T$ be a positive constant.

Given matrices $F(t), G(t), H(t), 0<t<T$, such that

$$
\left\{\begin{array}{l}
F(\cdot), G(\cdot) \in C\left([0, T] ; \mathbf{R}^{N} \times \mathbf{R}^{N}\right), \\
H(\cdot) \in C\left([0, T] ; \mathbf{R}^{N} \times \mathbf{R}^{P}\right)
\end{array}\right.
$$

Received by the editors November 20, 1979 and, in revised form, June 20, 1980.

AMS (MOS) subject classifications (1970). Primary 93E99, 60J70; Secondary 35J70, 93E10, 49G99, $60 \mathrm{~J} 60$.

Key words and phrases. Separation principle, filtering problems, quasi-variational inequalities, ellipticparabolic operators, impulse control problems, degenerate diffusions. 
we denote by $y^{\circ}(t)$ the solution of the linear Itô equation

$$
\left\{\begin{array}{l}
d y^{\circ}(t)=F(t) y^{\circ}(t) d t+G(t) d w(t), \quad 0<t<T, \\
y^{\circ}(0)=x+\zeta, \quad x \in \mathbf{R}^{N},
\end{array}\right.
$$

where $w(t)$ is a standard Wiener process in $\mathbf{R}^{N}$ and $\zeta$ is a Gaussian random variable with vanishing expectation and covariance matrix $P_{0} ; \zeta$ is independent of the process $w(t), 0<t<T$.

The current state of the system without control at the instant $t$ is $y^{\circ}(t)$, but we cannot observe the system. The information is provided by the channel output $z^{\circ}(t)$ defined by

$$
\left\{\begin{array}{l}
d z^{\circ}(t)=H(t) y^{\circ}(t) d t+d \eta(t), \quad 0<t<T, \\
z^{\circ}(0)=0,
\end{array}\right.
$$

where $\eta(t)$ is a Wiener process in $\mathbf{R}^{P}$ independent of $w(t)$, with vanishing expectation and covariance matrix $R(t)$ such that

$$
\left\{\begin{array}{l}
R(\cdot) \in C\left([0, T] ; \mathbf{R}^{P} \times \mathbf{R}^{P}\right), \\
R(t)>r I, \quad r>0 \quad \forall t \in[0, T] .
\end{array}\right.
$$

We denote by $\mathscr{Z}^{t}, 0<t<T$, the nondecreasing right continuous family of completed $\sigma$-algebras generating by the process $z^{\circ}(t)$.

An admissible impulse control $\nu$ is a set $\left\{\theta_{1}, \xi_{1} ; \ldots ; \theta_{i}, \xi_{i} ; \ldots\right\}$ where $\left\{\theta_{i}\right\}_{i=1}^{\infty}$ is an increasing sequence of stopping times with respect to $\mathscr{L}^{t}$ convergent to $T$ $\left(0<\theta_{i}<\theta_{i+1}<T,\left[\theta_{i}<t\right] \in \mathscr{Z}^{t}, \theta_{i} \rightarrow T\right)$ and $\left\{\xi_{i}\right\}_{i=1}^{\infty}$ is a sequence of random variables taking values in $\mathbf{R}_{+}^{N}$, adapted with respect to $\left\{\theta_{i}\right\}_{i=1}^{\infty}\left(\xi_{i}: \Omega \rightarrow \mathbf{R}^{N}, \xi_{i}>0\right.$, $\mathfrak{Z}^{\theta i}$-measurable).

Now we define the sequence of diffusion processes with jumps, $\left\{y^{n}(t)\right\}_{n-1}^{\infty}$, $y^{n}(t)=y^{n}(t, \nu), t \in[0, T], \nu$ any admissible impulse control, by the stochastic equation

$$
\left\{\begin{array}{l}
d y^{n}(t)=F(t) y^{n}(t) d t+G(t) d w(t), \quad \theta_{n}<t<T \\
y^{n}(t)=y^{n-1}(t)+1_{\theta_{n}-t} \xi_{n}, \quad 0<t<\theta_{n}{ }^{1}
\end{array}\right.
$$

We have

$$
y^{n}(t)=y^{i}(t) \text { on }\left[0, \theta_{n}[, \forall i>n\right.
$$

Defining

$$
y(t, \nu)=\lim _{n \rightarrow \infty} y^{n}(t), \quad 0<t<T,
$$

the process $y(t)=y(t, v)$, which is right continuous with left limits existing, satisfies the following stochastic equation:

$$
\left\{\begin{array}{l}
d y(t)=F(t) y(t) d t+G(t) d w(t)+\sum_{i=1}^{\infty} \xi_{i} \delta\left(t-\theta_{i}\right) d t, \quad 0<t<T, \\
y(0)=x+\zeta,
\end{array}\right.
$$

where $\delta(t)$ is the Dirac measure.

\footnotetext{
${ }^{1} 1_{\theta_{n}, t}$ denotes the characteristic function of the set $\left\{\theta_{n}=t\right\}$.
} 
The current state of the system with impulse control $\nu$ at the instant $t$ is represented by $y(t)$, and

$$
\hat{y}(t)=E\left\{y(t) / \mathscr{Z}^{t}\right\}
$$

is the information state process; we also have $\hat{y}(0)=x$.

We call the impulse process $\beta(t)$ the solution of the equation

$$
\left\{\begin{array}{l}
d \beta(t)=F(t) \beta(t) d t+\sum_{i=1}^{\infty} \xi_{i} \delta\left(t-\theta_{i}\right) d t, \quad 0<t<T, \\
\beta(0)=0 .
\end{array}\right.
$$

Clearly, $\beta(t)=\beta(t, \nu)$ is built in the same way as $y(t)$ by iteration. Notice, the process $\beta(t)$ is right continuous with left limits and adapted to the observation $\mathscr{Z}^{t}$. Thus, according to the equation (2.2), (2.8), (2.10) we deduce from (2.9)

$$
\hat{y}(t)=E\left\{y^{\circ}(t) / \mathscr{I}^{t}\right\}+\beta(t) \text {. }
$$

We introduce the process $\varepsilon(t)$, called the estimation error, given by

$$
\varepsilon(t)=y^{\circ}(t)-E\left\{y^{\circ}(t) / \mathscr{L}^{t}\right\}, \quad 0<t<T,
$$

which is independent of $\mathscr{Z}^{t}$ and verifies

$$
\varepsilon(t)=y(t)-\hat{y}(t), \quad 0<t<T .
$$

We also define $\hat{w}(t)$ by

$$
\left\{\begin{array}{l}
d \hat{w}(t)=R^{-1 / 2}(t) H(t) \varepsilon(t) d t+R^{-1 / 2}(t) d \eta(t), \quad 0<t \leqslant T, \\
\hat{w}(0)=0
\end{array}\right.
$$

which is a standard Wiener process and satisfies the martingale property

$$
\hat{w}(t)=E\{\hat{w}(s) / \mathscr{Z}\}, \quad 0<t<s<T .
$$

Then, the assertions (2.10), (2.11) and the R. E. Kalman-R. S. Bucy [4] theory show that $\hat{y}(t)$ is the solution of the following stochastic equation

$$
\left\{\begin{array}{lr}
d \hat{y}(t)=F(t) \hat{y}(t) d t+P(t) H^{*}(t) R^{-1 / 2}(t) d \hat{w}(t)+\sum_{i=1}^{\infty} \xi_{i} \delta\left(t-\theta_{i}\right) d t, & 0<t<T, \\
\hat{y}(0)=x, & (2.16)
\end{array}\right.
$$

where the matrix $P(t)$ is the unique solution of the Riccati equation

$$
\left\{\begin{array}{l}
P^{\prime}(t)=F P+P F^{*}-P H^{*} R^{-1} H P+G G^{*}, \quad 0<t<T, 2 \\
P(0)=P_{0} .
\end{array}\right.
$$

We also deduce that the estimation error $\varepsilon(t)$ is the unique solution of the Ito equation

$$
\left\{\begin{array}{l}
d \varepsilon(t)=\left(F-P H^{*} R^{-1} H\right) \varepsilon d t-P H^{*} R^{-1} d \eta+G d w, \quad 0<t<T, \\
\varepsilon(0)=\zeta .
\end{array}\right.
$$

\footnotetext{
2 The prime (') means time derivative and the star (*) denotes the transpose.
} 
3. Optimal impulse control. Let $f(x, t)$ be a nonnegative, continuous and bounded function on $\mathbf{R}^{N} \times[0, T]$ taking values in $\mathbf{R}$,

$$
f \in C_{b}\left(\mathbf{R}^{N} \times[0, T]\right), \quad f(x, t)>0 \forall x \in \mathbf{R}^{N}, t \in[0, T],
$$

and let $k(\xi)$ be a continuous function from $\mathbf{R}_{+}^{N}$ into $\mathbf{R}$ such that

$$
k \in C\left(\mathbf{R}_{+}^{N}\right), \quad k(\xi)>k_{0}>0, \quad k(\xi) \rightarrow \infty \quad \text { if }|\xi| \rightarrow \infty .
$$

Now, for any admissible impulse control $\nu=\left\{\theta_{1}, \xi_{1} ; \ldots ; \theta_{i}, \xi_{i} ; \ldots\right\}$ and $x \in \mathbf{R}^{N}$ we set

$$
J_{x}(\nu)=E\left\{\int_{0}^{T} f(y(t), t) e^{-\alpha t} d t+\sum_{i=1}^{\infty} k\left(\xi_{i}\right) 1_{\theta_{i}<T} e^{-\alpha \theta_{i}}\right\},
$$

where $\alpha$ is a real constant.

We remark that any admissible impulse control $\nu$ is adapted to the information state $\hat{y}(t)$ and not to the current state $y(t)$.

Our purpose is to characterize the optimal cost

$$
u_{0}(x)=\inf \left\{J_{x}(\nu) / \nu \text { admissible impulse control }\right\}
$$

and to obtain a separation principle for an eventual optimal admissible impulse control.

Let $M$ be the operator

$$
[M \phi](x)=\inf \left\{k(\xi)+\phi(x+\xi) / \xi \in \mathbf{R}_{+}^{N}\right\}
$$

and $u(x, t)$ be an arbitrary function satisfying

$$
u \in C_{b}\left(\mathbf{R}^{N} \times[0, T]\right), \quad u<M u \text { in } \mathbf{R}^{N} \times[0, T] .
$$

The admissible impulse control $\nu=\nu_{x}$ associated to the function $u$ is defined as follows. First we select a function $\xi(x, t)$ verifying

$$
\left\{\begin{array}{l}
\xi: \mathbf{R}^{N} \times[0, T] \rightarrow \mathbf{R}_{+}^{N}, \text { Borel measurable and bounded such that } \\
{[M u](x, t)=k(\xi(x, t))+u(x+\xi(x, t), t) \quad \forall x \in \mathbf{R}^{N}, t \in[0, T] .}
\end{array}\right.
$$

Next, define $\tilde{\theta}^{\circ}=0$ and $\hat{y}^{\circ}(t)$ by

$$
\left\{\begin{array}{l}
d \hat{y}^{\circ}(t)=F(t) \hat{y}^{\circ}(t) d t+P(t) H^{*}(t) R^{-1 / 2}(t) d \hat{w}(t), \quad 0<t<T, \\
\hat{y}^{\circ}(0)=x .
\end{array}\right.
$$

We define $\nu=\left\{\theta_{1}, \xi_{1} ; \ldots ; \theta_{i}, \xi_{i} ; \ldots\right\}$ by the formulas

$$
\begin{gathered}
\tilde{\theta}^{i+1}=\inf \left\{t \in\left[\tilde{\theta}^{i}, T\right] / u\left(\hat{y}^{i}(t), t\right)=[M u]\left(\hat{y}^{i}(t), t\right)\right\}, \quad i=0,1, \ldots, \\
\theta_{i}= \begin{cases}\tilde{\theta}^{i} & \text { if } \tilde{\theta}^{i}<T, i=1,2, \ldots, \\
T & \text { otherwise, }\end{cases} \\
\xi_{i}=\xi\left(\hat{y}^{i-1}\left(\theta_{i}\right), \theta_{i}\right), \quad i=1,2, \ldots, \\
\left\{\begin{array}{cc}
d \hat{y}^{i}(t)=F(t) \hat{y}^{i}(t) d t+P(t) H^{*}(t) R^{-1 / 2}(t) d \hat{w}(t), \\
\theta_{i}<t<T,
\end{array}\right. \\
\hat{y}^{i}(t)=\hat{y}^{i-1}(t)+1_{\theta_{i}-t}, \quad 0<t<\theta_{i} .
\end{gathered}
$$


Clearly, if there exists a function $u$ verifying (3.6) whose associated admissible impulse control $\nu$ is optimal, the separation principle is established. Notice, the fact that $\nu$ is optimal shows automatically that $\theta_{i} \rightarrow T$. Moreover, $\theta_{i}=T$ for all $i>n(\omega)$ almost surely.

Let $A(t)$ be the second order differential operator corresponding to the Itô equation (3.8),

$$
A(t)=-\sum_{i, j=1}^{N} a_{i j}(t) \frac{\partial^{2}}{\partial x_{i} \partial x_{j}}-\sum_{i=1}^{N}(F(t) x)_{i} \frac{\partial}{\partial x_{i}}+\alpha,
$$

where

$$
\left[a_{i j}(t)\right]_{i j}=\frac{1}{2} P(t) H^{*}(t) R^{-1}(t) H(t) P(t) .
$$

We remark that $A(t)$ is usually degenerate. W. M. Wonham [8], M. H. A. Davis [3], A. Bensoussan and J. L. Lions [1] supposed that the matrices $P(t)$ and $H(t)$ are nonsingular.

We set

$$
l(x, t)=E\{f(x+\varepsilon(t), t)\} \quad \forall x \in \mathbf{R}^{N}, t \in[0, T],
$$

where $\varepsilon(t)$ is given by (2.18).

We introduce the following quasi-variational inequality. Find $u(x, t)$ such that

$$
\left\{\begin{array}{l}
u \in C_{b}\left(\mathbf{R}^{N} \times[0, T]\right), \quad u(x, T)=0 \forall x \in \mathbf{R}^{N}, \\
-\frac{\partial u}{\partial t}+A(t) u<l \quad \text { in } \mathscr{D}^{\prime}\left(\mathbf{R}^{N} \times[0, T]\right), u<M u \text { in } \mathbf{R}^{N} \times[0, T] \\
-\frac{\partial u}{\partial t}+A(t) u=l \quad \text { in } \mathscr{D}^{\prime}([u<M u]) .^{3}
\end{array}\right.
$$

We have the

Separation Principle TheOrem. Let the assumptions (2.1), (2.4), (3.1), (3.2) hold. Then there exists one and only one solution $u$ of the quasi-variational inequality (3.16). Moreover the admissible impulse control $\nu$ defined by (3.7)-(3.12), associated to the function $u$ given by (3.16), is optimal [i.e., $\left.u_{0}(x)=J_{x}\left(\nu_{x}\right)\right]$.

Proof. First, using a general result in [6] applied to a degenerate operator $-\partial / \partial t+A(t)$, we deduce that there exists a solution of problem (3.16).

In order to prove the uniqueness, we denote by $z(s)=z_{x t}(s, \omega), 0<t<s<T$, $x \in \mathbf{R}^{N}, \omega \in \Omega$, the diffusion associated to the operator $-\partial / \partial t+A(t)$, i.e.,

$$
\left\{\begin{array}{l}
d z(s)=F(s) z(s) d s+P(s) H^{*}(s) R^{-1 / 2}(s) d \hat{w}(s), \quad t<s<T, \\
z(t)=x .
\end{array}\right.
$$

Now let $u(x, t)$ be an arbitrary solution of (3.16). We set $\theta=\theta_{x t}(\omega), 0<t<T$, $x \in \mathbf{R}^{N}, \omega \in \Omega$, the first exit time of process $z(s)$ from $[u<M u]$, i.e.,

$$
\theta=\inf \{s \in[t, T] / u(z(s), s)=[M u](z(s), s)\}
$$

\footnotetext{
${ }^{3} C_{b}$ denotes the space of continuous and bounded functions, and $\Phi '$ is the space of distributions.
} 
Then, using the fact that the coefficients of the second order terms of operator $A(t)$ are constant and that $u(x, t)$ is continuous, we establish by convolution techniques the following Itô formulas for each $x \in \mathbf{R}^{N}, t \in[0, T]$ :

$$
\begin{array}{r}
u(x, t)<E\left\{\int_{t}^{T \wedge \tau} l(z(s), s) e^{-\alpha s} d s+u(z(T \wedge \tau), T \wedge \tau) e^{-\alpha(T \wedge \tau)}\right\} \\
\forall \tau>t \text { stopping time, } \\
u(x, t)=E\left\{\int_{t}^{\theta} l(z(s), s) e^{-\alpha s} d s+u(z(\theta), \theta) e^{-\alpha \theta}\right\}
\end{array}
$$

Therefore, as in [6], the properties (3.19), (3.20) imply the uniqueness of the solution $u$.

Next, from (3.7)-(3.12) and (3.20), we deduce

$$
u(x, 0)=E\left\{\int_{0}^{T} l(\hat{y}(t), t) e^{-\alpha t} d t+\sum_{i=1}^{\infty} k\left(\xi_{i}\right) 1_{\theta_{1}<T} e^{-\alpha \theta_{1}}\right\},
$$

and from (2.13), (3.15) we have

$$
E\left\{\int_{0}^{T} l(\hat{y}(t), t) e^{-\alpha t} d t\right\}=E\left\{\int_{0}^{T} f(y(t), t) e^{-\alpha t} d t\right\}
$$

hence

$$
u(x, 0)=J_{x}(\nu), \quad \nu \text { associated to } u .
$$

Similarly, using (3.19), we obtain

$$
u(x, 0)=\inf \left\{J_{x}(\nu) / \nu \text { admissible impulse control }\right\} .
$$

Then, (3.23) and (3.24) give

$$
u(x, 0)=u_{0}(x) \text {, optimal cost (3.4), }
$$

and the theorem is proved.

RemarK 1. If the function $f(x, t)$ is Lipschitz continuous, so is the function $u(x, t)$. In this case, $u$ is also the maximum solution of a classical quasi-variational inequality introduced by A. Bensoussan and J. L. Lions [2].

Remark 2. This result can be extended for a function $k(\xi, x, t)$ instead of $k(\xi)$ appearing in the definition of cost (3.3). Clearly, we can replace the condition $\xi \in \mathbf{R}_{+}^{N}$ by $\xi \in \Lambda$, where $\Lambda$ is a closed subset of $\mathbf{R}^{N}$.

REMARK 3. Using the technique presented in this paper, we can improve the result obtained in [5].

\section{REFERENCES}

1. A. Bensoussan and J. L. Lions, Applications des inéquations variationnelles en contrôle stochastique, Dunod, Paris, 1978.

2. Contrôle impulsionnel et inéquations quasi-variationnelles, Dunod, Paris, 1979.

3. M. H. A. Davis, The separation principle in stochastic control via Girsanov solutions, SIAM J. Control Optim.14 (1976), 176-188.

4. R. E. Kalman and R. S. Bucy, New results in linear filtering and prediction theory, Trans. ASME Ser. D J. Basic Engrg. 83 (1961), 95-108. 
5. J. L. Menaldi, Le principe de séparation pour le problème de temps d'arrêt optimal, Stochastics 3 (1979), 47-59.

6. On the optimal impulse control problem for degenerate diffusions, SIAM J. Control Optim. 18 (1980), 722-739.

7. J. Szpirglas and G. Mazziotto, Théorème de séparation pour l'arrêt optimal, Lecture Notes in Math., vol. 721, Springer-Verlag, Berlin and New York, 1979.

8. W. M. Wonham, On the separation theorem of stochastic control, SIAM J. Control 6 (1968), 312-326.

Department of Mathematics, University of Kentucky, LeXington, Kentucky 40506

Current address: I.N.R.I.A., Boite Postale 105, 78153 Le Chesnay Cedex, France 